\title{
Synthesis of Spiked Plasmonic Nanorods with an Interior Nanogap for Quantitative Surface-Enhanced Raman Scattering Analysis
}

\author{
Yang Zhang, ${ }^{\dagger}$ Chen Li, ${ }^{\ddagger}{ }^{\ddagger}$ Zahra Fakhraai, ${ }^{\ddagger \odot}$ Basem Moosa, ${ }^{\dagger}$ Peng Yang, ${ }^{\dagger}$ and Niveen M. Khashab ${ }^{*}{ }^{\dagger}$ Pe \\ ${ }^{\dagger}$ Smart Hybrid Materials (SHMs) Laboratory, Advanced Membranes and Porous Materials Center, King Abdullah University of \\ Science and Technology (KAUST), Thuwal 23955-6900, Kingdom of Saudi Arabia \\ ${ }^{\ddagger}$ Department of Chemistry, University of Pennsylvania, Philadelphia 19104, United States
}

Supporting Information

\begin{abstract}
Realizing quantitative surface-enhanced Raman scattering (SERS) analysis is extremely helpful and challenging. Here, we utilize a facile method to synthesize spiked plasmonic nanorods with an interior gap. The Raman signal from the molecules embedded in the gap can be dramatically enhanced, leading to strong, stable, and reproducible SERS signals that can be used as an internal reference for quantitative SERS analysis. We demonstrate that the rough exterior surface has a good performance in enhancing the Raman signal of polycyclic aromatic hydrocarbon molecules adsorbed on the surface. The result shows that this method is applicable for a large range of analyte concentrations and there is an excellent linear relationship between the SERS intensity ratio and the analyte concentration $(0.5-100 \mu \mathrm{M})$.
\end{abstract}

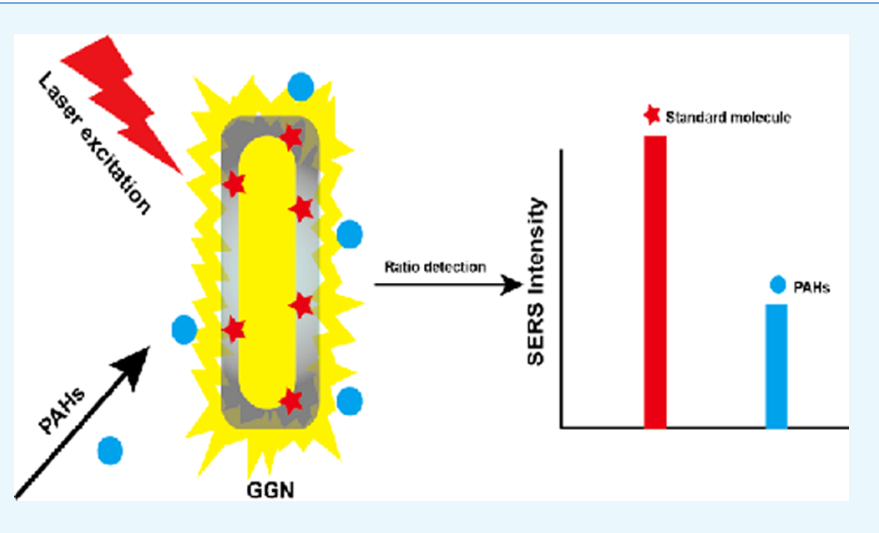

\section{INTRODUCTION}

Surface-enhanced Raman scattering (SERS) has been recognized as a powerful protocol that is capable of detecting molecular vibrations, which can be viewed as fingerprint information on various organic molecules, biomolecules, and cells and tissues. ${ }^{1-3}$ SERS is an ultrasensitive method, as the Raman signal can be dramatically enhanced as much as $10^{10}$ to $10^{11}$ times in some plasmonic nanostructure, ${ }^{4}$ which allows to detect even one single molecule. ${ }^{4-7}$ Compared with the conventional techniques such as fluorescence, electrochemistry, and high-performance liquid chromatography, SERS holds significant advantages. $^{8-12}$ For example, higher resolution on multiplex samples as SERS provides fingerprint signatures of analytes endowing with better anti-interference resistance. Generally, SERS enhancement is ascribed to electromagnetic and chemical enhancement, and the former contributes to most of SERS enhancement. The electromagnetic enhancement is localized at the "hotspots" resulting from the coupling of the localized surface plasmon resonance (LSPR) on the surface or in the junctions of plasmonic nanoparticles. ${ }^{13-16}$ Optimizing the structure of plasmonic nanoparticles including size, shape, surface morphology, and composition is important in improving the reproducibility and sensitivity of SERS assays. The typical approach is to use the target molecule to induce the aggregation of nanoparticles forming a large number of hotspots in solution. ${ }^{8,17}$ However, problems still remain due to the random and poor reproducibility of the hotspots, resulting in lack of quantitative SERS data and wide distribution of enhancement factors. ${ }^{13}$ In addition, the operational and instrumental factors also have a profound influence on the Raman signal. ${ }^{17}$ Therefore, to date, it is still a great challenge to realize quantitative SERS analysis. ${ }^{18-20}$

In previous studies, researchers have attempted to use internal standards to achieve sensitivity and reproducibility simultaneously. ${ }^{21-26}$ However, the internal standard molecules can be influenced by the microenvironment of the solution and compete for the surface adsorption, leading to the fluctuation of the intensity and frequency. ${ }^{1,27,28}$ Recently, a new structure based on core-molecule-shell nanoparticles has captured great attention. ${ }^{4,29,30}$ The interior gap between the core and the shell provides controllable hotspots and uniformity for SERS enhancement. ${ }^{4,31}$ The Raman molecules inside the gap could generate a highly stable, strong, and quantitative SERS signal. In particular, DNA, polymer-functionalized spherical Au nanoparticles, and galvanic replacement reaction have been reported to synthesize these nanostructures with nanometer interior gaps, exhibiting strong and stable SERS signals. ${ }^{4,29,30,32,33}$ For example, it was shown that galvanic replacement reaction between silver and gold facilitates the formation of gold nanorods (GNRs) with uniform interior nanogaps with a stable, strong, and reproducible SERS signals. ${ }^{32}$ Other methods such as small molecule-interlayered plasmonic structure and $\mathrm{SiO}_{2}$ interlayered gold structures also have been used to synthesize nanostructures with interior

Received: $\quad$ May 27, 2018

Accepted: August 2, 2018

Published: October 30, 2018 
gaps. ${ }^{34-36}$ The standard molecule inside the gap between the core and shell was in a safe environment without perturbation from outside, and the surface of the shell was free without competitive adsorption. However, for these structures, they still have some shortcomings, such as laborious synthetic procedures and long preparation times. More importantly, all of these structures reported so far have smooth surfaces. It has been shown that the most important factor affecting SERS intensity is the shape of the nanoparticles. ${ }^{37}$ The shapes with tips and edges can localize the plasmonic near field and create hotspots around the edges. ${ }^{38-40}$ The plasmonic nanostructures with more tips and edges will provide higher sensitivity in SERS analysis. ${ }^{41,42}$ Up to now, there has been no method to synthesize such spiked plasmonic nanorods with an interior gap, without the need to use DNA templates, for direct quantitative SERS analysis. Herein, an efficient method to prepare plasmonic substrates with spiked surfaces and interior gaps by employing a polydopamine internal shell is reported. This structure is very reliable and suitable for quantitative SERS analysis, as the spiked surface provides a higher surface enhancement in a variety of samples. We used 4-mercaptopyridine (4-mp) as an internal standard in the nanogap (Scheme 1 ). This is a label-free method with high sensitivity and was

Scheme 1. Schematic Illustration of the Synthesis of the Plasmonic Core-Shell Structure, Based on GNRs, for Quantitative SERS Analysis of PAHs

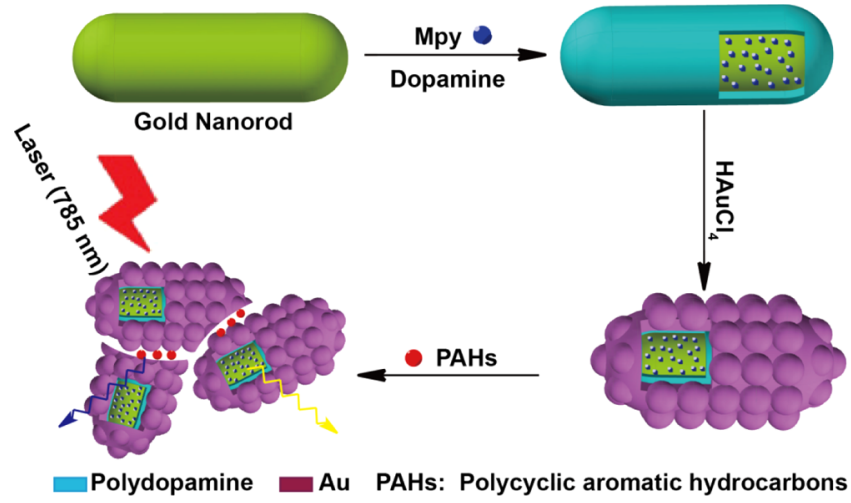

successfully tested to detect polycyclic aromatic hydrocarbons (PAHs) quantitatively. The limit of detection is up to $0.3 \mu \mathrm{M}$. Finite-difference time-domain (FDTD) simulation is also used to confirm these results.

\section{RESULTS AND DISCUSSION}

Only three steps are needed to synthesize the GGN with interior gaps. Herein, we introduced pristine GNRs as the core (Figure S1), of which the surface was modified by 4-mp as internal molecules via $\mathrm{Au}-\mathrm{S}$ bonds. Afterward, the mpGNRs were treated with dopamine (DA) and tris buffer $(\mathrm{pH}=8)$ for $1 \mathrm{~h}$ to yield poly-DA-coated species (mpGNRs@DA). Noted that, under alkaline conditions, phenolic catechols were oxidized to quinones, causing the formation of 5,6-dihydroxyindolines, their derivatives, and then leading to the polymerization to form oligomers. These were crammed by $\pi-\pi$ stacking, charge transfer, and hydrogen bonding to form a nanometer thick poly-DA layer. ${ }^{43}$ As shown in Figure S2, we can see that there is a thin layer of poly-DA outside the GNRs and the thickness is about $2.6 \mathrm{~nm}$. These functional groups such as catechol, amine, and imine can serve as both an anchor and reaction sites for loading metal ions, ${ }^{44,45}$ and the catechol takes the role of a reducing agent for gold nucleation and growth. ${ }^{46}$ Catechol was oxidized to quinones by $\mathrm{Au}(\mathrm{III})$, and small gold nanoparticles were produced on randomly oriented oxidized sites of the poly-DA shell. Further anisotropic growth of gold nanoparticles were activated by the reduction of $\mathrm{HAuCl}_{4}$ by hydroxyl amine which was subsequently added. ${ }^{10}$ From the transmission electron microscopy (TEM) and scanning electron microscopy images (Figures 1a and S3),

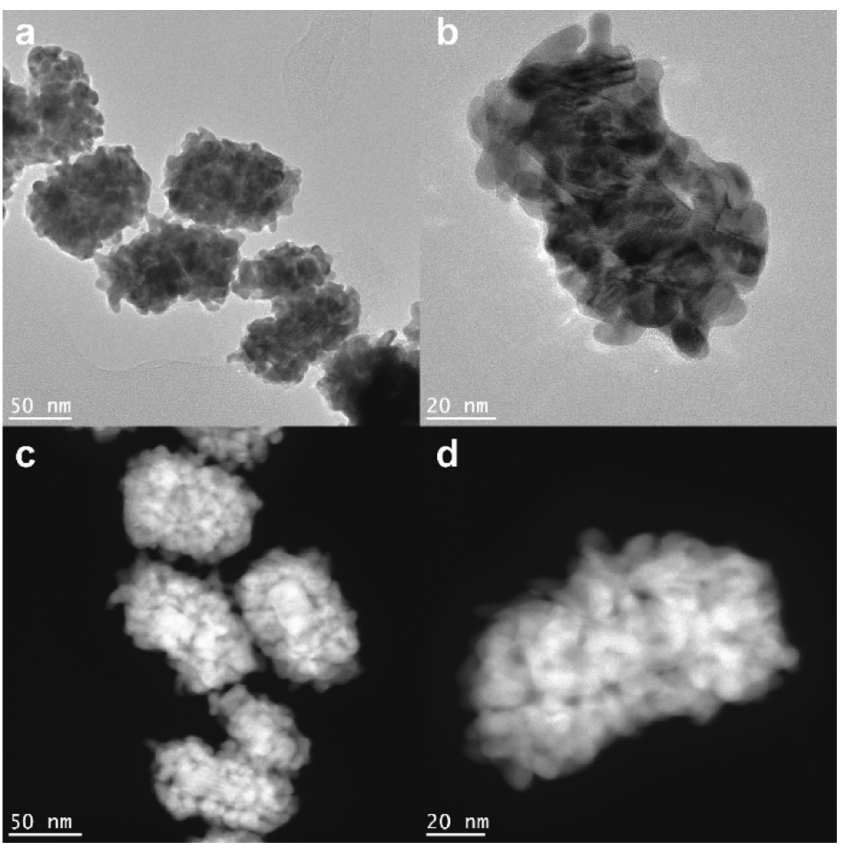

Figure 1. (a) TEM image of GGN with interior gaps. (b) Highresolution transmission electron microscopy image of a typical GGN. (c) STEM image of GGN with interior gaps. (d) STEM image a typical GGN.

we found that the GGNR structure is uniform and the surface of gold shell is rough and spiky. We applied scanning TEM (STEM) to demonstrate the core-shell structure with an interior gap, as expect, the spatial distribution of the Au core, the spiked gold shell, and the interior gap could be clearly identified (Figure $1 \mathrm{c}, \mathrm{d}$ ). It is worth mentioning that the amount of $\mathrm{HAuCl}_{4}$ plays an important role in the synthesis. The density of the small gold nanoparticles increases by raising the amount of $\mathrm{HAuCl}_{4}$ (Figure S4). At first, there are some sporadic gold nanoparticles on the surface; then, it becomes a whole shell. In case more $\mathrm{HAuCl}_{4}$ added, the particles will become bigger and smooth (Figure S4c).

The SERS properties of GGN were tested by three laser colors $(785,660$, and $532 \mathrm{~nm}$ ) (Figure S5). Excited by $785 \mathrm{~nm}$ laser, GGN shows the most glaring response in the near infrared region. In the case of $532 \mathrm{~nm}$, little SERS signal was observed, by contrast to 785 or $660 \mathrm{~nm}$, where the SERS signals were raised enormously. This is because $532 \mathrm{~nm}$ is outside the peak region of the UV-vis spectrum of nanorods (Figure S6). Under laser irradiation, there is no measurable changes in time sequence SERS spectra of GGN (Figure S7). This leaves out the laser trapping effect on the aggregation of particles. ${ }^{1,47}$ From these experimental results, sufficient and stable SERS signals were obtained with excitation at either 785 $\mathrm{nm}$ or $660 \mathrm{~nm}$. 

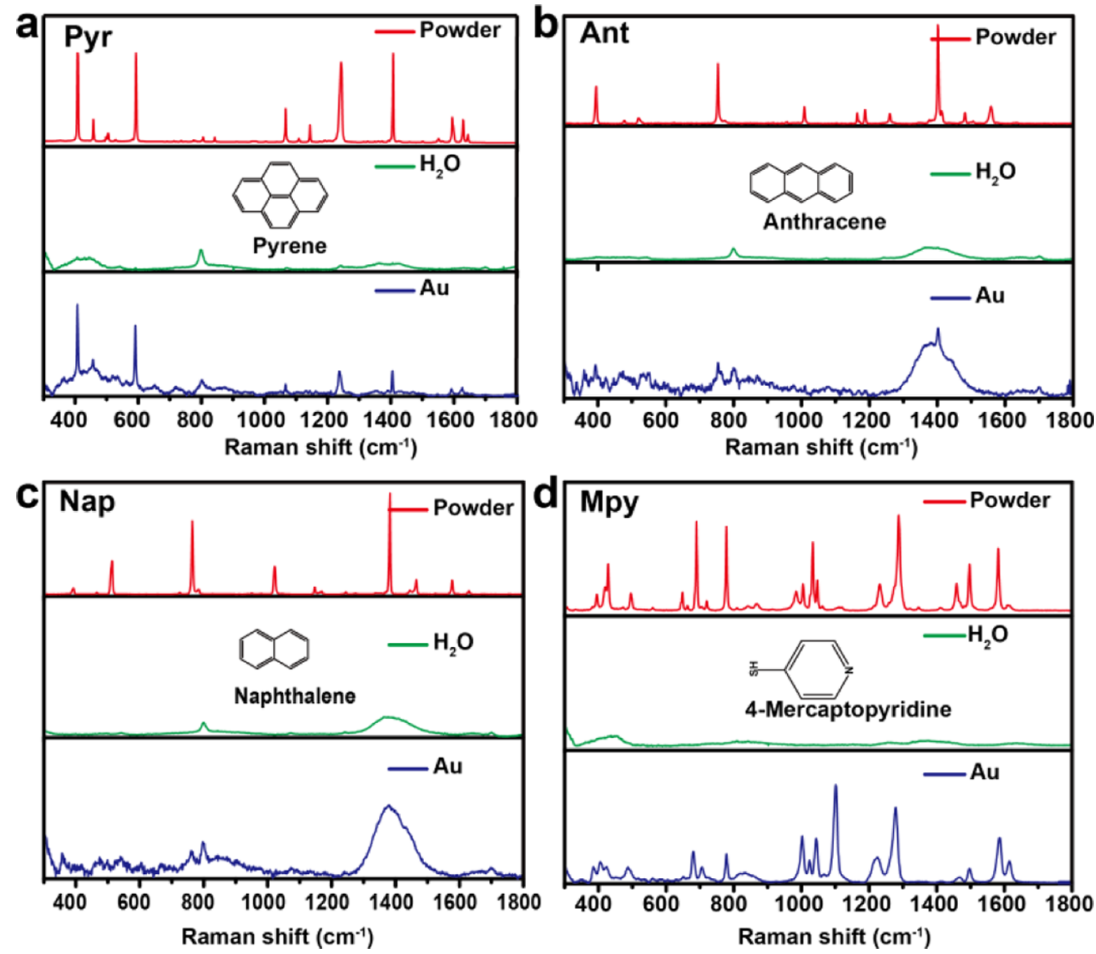

Figure 2. Raman spectra of Pyr (a), Ant (b), Nap (c), and 4-mp (d) powder, in water and mixed with GNRs. The spectra were obtained by 785 nm laser.

We used pyrene (Pyr), anthracene (Ant), and naphthalene (Nap) as target molecules that are PAHs to verify the quantitative SERS analysis. The PAH molecules can be adsorbed on the surface of GGN. In the adsorption process, the time-dependent SERS spectra exhibited stable signals in 1 $\mathrm{h}$ after the addition of PAH molecules (Figure S8). The Pyr, Ant, and Nap all have a few characteristic peaks as compared to 4-mp, and the unique peaks for 4-mp are located around $\sim 1000 \mathrm{~cm}^{-1}$ (Figure 2). It has been experimentally proved that nearly no Raman signals of Pyr, Ant, Nap, and 4-mp in water were found even at a high concentration level $\left(3 \times 10^{-4} \mathrm{M}\right)$. However, the SERS signals of Pyr, Ant, Nap, and 4-mp mixed GNRs were remarkably enhanced (Figure 2). As a result, it could be concluded that the SERS signals were generated as these molecules reside close to the particle surfaces but not from the molecules themselves during the detection.

Moreover, the as-synthesized GGN was mixed with Pyr of various concentrations for quantitative SERS analysis. After incubation of $1 \mathrm{~h}$ with the addition of Pyr, two new peaks of Pyr have been observed at $\sim 590$ and $\sim 1400 \mathrm{~cm}^{-1}$. The SERS intensity of Pyr fluctuated with parallel samples, but the reproducibility can be improved by normalizing the data to that of 4-mp (at $\sim 1000 \mathrm{~cm}^{-1}$ ) (Figure S9). By lowering the concentration, the intensity of the characteristic peaks of Pyr declines correspondingly (Figure 3a). In order to correct the fluctuations in the instrumental factors and other unknown factors, the Pyr signals (at $\sim 590 \mathrm{~cm}^{-1}$ ) were normalized to the SERS intensity of internal molecules $4-\mathrm{mp}\left(\right.$ at $\sim 1000 \mathrm{~cm}^{-1}$ ). The intensity ratio of the target molecule (Pyr) to internal standard molecule shows a good linear relationship with the Pyr concentration (Figure 3b). The same operation was used for Ant and Nap. As depicted in Figure 3c, we can find that the peak intensities at $\sim 400, \sim 750$, and $\sim 1400 \mathrm{~cm}^{-1}$ are all decreasing along with the concentration of Ant going down; the normalized peak at $\sim 1400 \mathrm{~cm}^{-1}$ to that of 4-mp was used for quantitative detection. The relative SERS intensity versing the Ant concentration follows a very nice curve (Figure 3d). We further applied the GNN to the quantitative detection of Nap (Figure 3e,f). The linearity of these working cures demonstrated a larger range of the analyte concentration, where the highest concentration is 100 times larger than the lowest concentration. From the linear working curve, the minimum response value of Pyr, Ant, and Nap is lower than that has been reported in detecting $\mathrm{PAH}$ molecules. ${ }^{48,49}$

To better understand the optical properties of the nanorod structures and verify the role of roughness through the far-field LSPR peak on the wavelengths with observed Raman enhancements, we performed UV-vis spectroscopy and compared our data with FDTD simulations for GGN. Figure 4a shows the UV-vis spectra as compared with the calculated far-field extinction cross sections of the two nanoparticles obtained by single-particle FDTD simulations. The calculated far-field extinction cross-sectional spectra for GGN were calculated for two polarization directions, along the long axis of the rod (longitudinal) and normal to the long axis (transverse). The larger extinction cross section at this wavelength for GNN contributes to the larger enhancement factor measured for these particles in Table 1. To explore the role of the roughness and extinction cross sections in the high performance of GGN as a SERS substrate, the near-field distribution around the modeled nanorod structures was calculated. Near-field enhancement contributes to Raman enhancement, and resonant optical extinction seen from the far field creates the strongest near-field enhancement, so we calculate near field at that wavelength. It has been reported that the SERS enhancement factor can be estimated from a term defined as the product of the electric near-field intensity at the excitation and Stokes frequencies. ${ }^{50-54}$ Figure $4 \mathrm{~b}$ shows the 

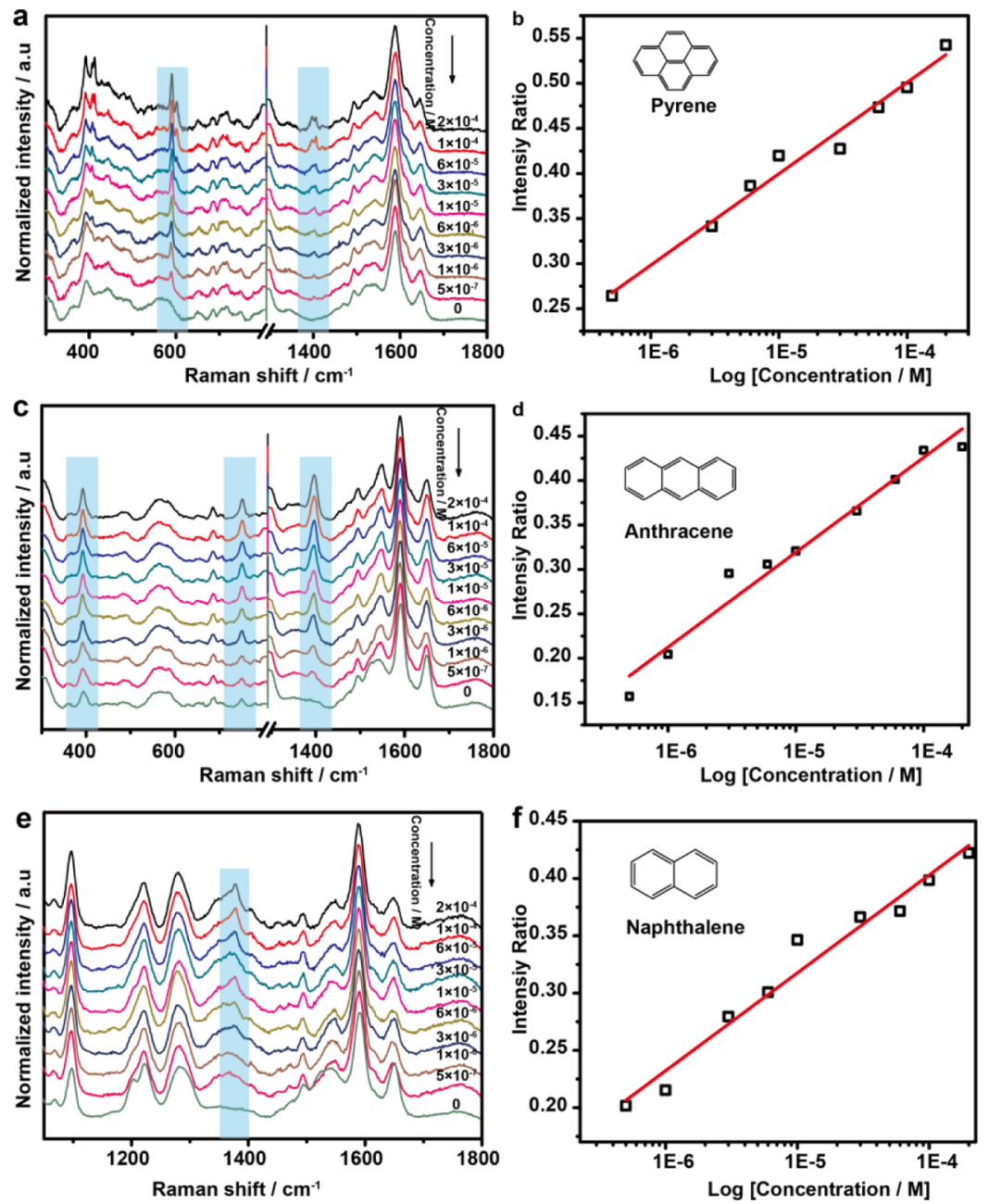

Figure 3. SERS spectra of GGN mixed with different concentrations of Pyr (a), Ant (c), and Nap (e) as well as the linear relationship of SERS intensities vs different concentrations of Pyr (b), Ant (d), and Nap (f). The spectra were obtained by $785 \mathrm{~nm}$ laser.
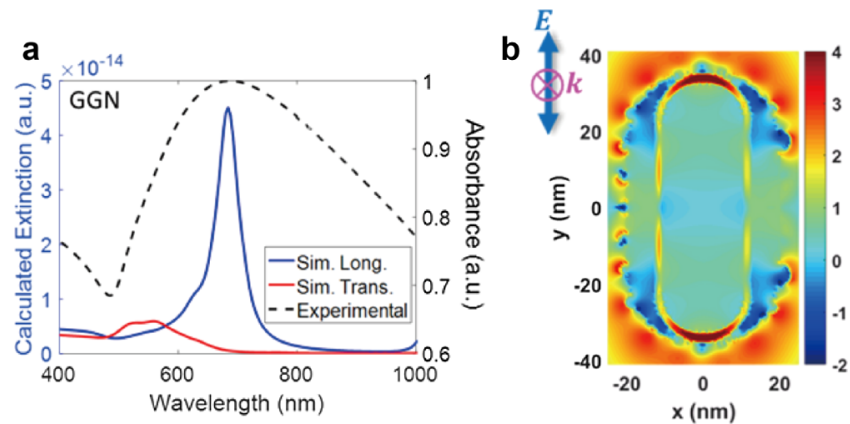

Figure 4. FDTD simulation results for a single simulated GGN structure. (a) Calculated far-field extinction spectra under longitudinal (blue) and transverse (red) incident polarizations, in comparison with experimentally measured UV-visible spectra (black, right axis). (b) Calculated near-field $\left(I_{\text {exc }} I_{\text {sto }}\right)$ distribution around the modeled nanorod at a Raman shift of $600 \mathrm{~cm}^{-1}$. The value at each position was calculated as the product of the electric field intensity at the excitation $(785 \mathrm{~nm})$ and Stokes $(824 \mathrm{~nm})$ wavelength.

calculated near-field distribution for an excitation wavelength of $785 \mathrm{~nm}$ and a Stokes wavelength of $824 \mathrm{~nm}$ (corresponding to a $600 \mathrm{~cm}^{-1}$ Raman shift) around a modeled GGN under longitudinal excitation. It can be seen from this structure that the plasmon resonance creates hotspots both inside the gap
Table 1. Total Values at All Raman Wavelengths Around a Modeled GGNR Structure and the Relative Contributions from Two Regions with Strong Hotspots Observed ${ }^{a}$

\begin{tabular}{cccc} 
& \multicolumn{3}{c}{ GGN } \\
\cline { 2 - 4 } $\begin{array}{c}\text { Stokes wavelength }(\mathrm{nm}) \\
\text { (Raman shift) }\end{array}$ & $\begin{array}{c}\text { total } E^{4} \\
\left(I_{\text {exc }} I_{\text {sto }}\right)\end{array}$ & $\begin{array}{c}\text { from outer } \\
\text { surface }\end{array}$ & $\begin{array}{c}\text { from inner } \\
\text { gap }\end{array}$ \\
$824\left(600 \mathrm{~cm}^{-1}\right)$ & 38.69 & 30.86 & 7.83 \\
$852\left(1000 \mathrm{~cm}^{-1}\right)$ & 33.00 & 24.73 & 8.27 \\
$882\left(1400 \mathrm{~cm}^{-1}\right)$ & 29.86 & 20.43 & 9.43
\end{tabular}

${ }^{a}$ The total was calculated as the summation over the volume within a smooth sphere-ended cylinder $2 \mathrm{~nm}$ outside the outer surface of the modeled nanorod. All values have the unit of $10^{7} \mathrm{~V}^{4} / \mathrm{m}^{4}$.

layer and at the shell surface. Whereas the gap hotspots appear to be stronger in intensity, the surface hotspots occupy a larger area, which also greatly affects the measured SERS enhancement. To quantify the relative contributions from these two types of hotspots, summation of the value over the corresponding regions was evaluated and shown in Table 1. This overall enhancement in the detection ability of GGN is consistent with the experimental observation of the higher sensitivity. 


\section{CONCLUSIONS}

We have designed and synthesized a new spiked plasmonic nanorod with an interior nanogap for quantitative SERS analysis by using an easy and cheap protocol. We used GNR as a core, 4-mp as an internal standard to develop a novel spiked rodlike core-molecule-shell nanostructure (GGN) with an interior gap by the support of polydopamine. Because of both closeness to the LSPR peak of the excitation wavelength and the rough surface, GGN provides a reliable SERS quantitative analysis in detecting PAH molecules, showing a strong potential as quantitative SERS detectors. The interior gap and surface roughness exhibited high SERS enhancements that were also confirmed by FDTD simulations. This will provide a new material for reliable quantitative SERS analysis, detection, Raman imaging, and photothermal therapy.

\section{EXPERIMENTAL SECTION}

Methods. $\mathrm{HAuCl}_{4} \cdot x \mathrm{H}_{2} \mathrm{O}$, hydroxylamine hydrochloride, polyvinyl pyrrolidone (PVP, $\left.M_{\mathrm{w}}=29000\right)$, hexadecyl trimethyl ammonium bromide (CTAB), $\mathrm{NaBH}_{4}$, ascorbic acid, 4-mp, DA, $\mathrm{AgNO}_{3}, \mathrm{H}_{2} \mathrm{SO}_{4}$, pyrene, anthracene, naphthalene, and tris(hydroxymethyl)aminomethane (Tris) were all purchased from Sigma-Aldrich and were used as received without further purification. The deionized (DI) water (Millipore Milli- $Q$ grade) prepared in-house, with a resistivity of $18.2 \mathrm{M} \Omega$, was used in all experiments. TEM images were taken with a FEI Titan 80-300 KV STEM (voltage $300 \mathrm{kV}$ ). The nanoparticles were dispersed in water and dropcasted on carbon-coated $\mathrm{Cu}$ grids. Raman spectra were collected by a Raman spectrometer (Horiba Jobin Yvon, Labram Aramis) at the respective excitation wavelength of 785, 660 , and $532 \mathrm{~nm}$. All of the Raman spectra of materials are collected in the liquid phase.

Synthesis of GNRs. The GNRs were synthesized via a seed-mediated procedure. Solution A: the gold seed solution was prepared by first mixing an aqueous solution of CTAB (7.5 $\mathrm{mL}, 0.1 \mathrm{M})$ and $\mathrm{HAuCl}_{4}(100 \mu \mathrm{L}, 24 \mathrm{mM})$. A freshly prepared aqueous solution (ice-cold) of $\mathrm{NaBH}_{4}(0.6 \mathrm{~mL}, 0.01 \mathrm{M})$ was then added to the above mixture, of which the color changed from yellow to brown. Then, $1.2 \mathrm{~mL}$ of $\mathrm{DI}$ water was further added, and the mixture was aged for 3-4 h. Solution B: an aqueous solution of ascorbic acid $(800 \mu \mathrm{L}, 0.1 \mathrm{M})$ was added to a mixed aqueous solution of $\mathrm{HAuCl}_{4}(2 \mathrm{~mL}, 24 \mathrm{mM})$, $\mathrm{H}_{2} \mathrm{SO}_{4}(2 \mathrm{~mL}, 0.5 \mathrm{M})$, CTAB $(100 \mathrm{~mL}, 0.1 \mathrm{M})$, and $\mathrm{AgNO}_{3}$ $(700 \mu \mathrm{L}, 10 \mathrm{mM})$, of which the color was changed from orange to colorless. For the final step, $240 \mu \mathrm{L}$ of solution A was added to solution B and kept at $30{ }^{\circ} \mathrm{C}$ for $12 \mathrm{~h}$.

Synthesis of 4-mp-Modified GNRs (mpGNRs). The prepared GNR solution ( $5 \mathrm{~mL}$ ) and an aqueous solution of 4$\mathrm{mp}\left(3.85 \times 10^{-4} \mathrm{M}, 1 \mathrm{~mL}\right)$ were mixed and stirred overnight.

Synthesis of DA-Coated mpGNRs (mpGNRs@DA). The mpGNRs@DA nanoparticles were synthesized as below: 1.5 $\mathrm{mL}$ of mpGNR solution was centrifuged and dispersed in the DA solution $(1.9 \mathrm{~mL}, 516 \mu \mathrm{M})$. A $0.5 \mathrm{~mL}$ of Tris $(10 \mathrm{mM})$ was then added. The mixture was sonicated for $1 \mathrm{~h}$. Finally, the mixture was centrifuged and resuspended in $1.5 \mathrm{~mL}$ of DI water.

Synthesis of Core-Shell Gold-Coated mpGNRs@DA (GGN). For the synthesis of GGN, $0.5 \mathrm{~mL}$ of mpGNRs@DA was diluted to $2.5 \mathrm{~mL}$ by adding $2 \mathrm{~mL}$ of DI water. $\mathrm{HAuCl}_{4}$ (150 $\mu \mathrm{L}, 5 \mathrm{mM})$, PVP $(50 \mu \mathrm{L}, \mathrm{w} / \mathrm{w}, 5 \%)$, and hydroxyl amine $(150 \mu \mathrm{L}, 50 \mathrm{mM})$ solutions were added consecutively. The mixture was then sonicated for $5 \mathrm{~min}$. Noted that, in order to explore the density of gold shell, different amounts of $\mathrm{HAuCl}_{4}$ $(5 \mathrm{mM}, 75$ and $250 \mu \mathrm{L})$ and hydroxyl amine $(50 \mathrm{mM}, 75$ and $250 \mu \mathrm{L})$ were tested.

FDTD Simulations. FDTD simulations on the optical properties of the single nanostructure were performed with Lumerical FDTD Solutions software v8.11. A typical GGN structure was modeled but with an additional layer of spheretipped gold nanocones to mimic the surface roughness (Figure S10). A 2.66 fs broadband total-field scatter-field pulse was used to excite the modeled structures with a boundary condition of perfectly absorbed layer applied to all directions. Detailed dimensions are shown in Table S1. All simulations were performed with a mesh size of $0.5 \mathrm{~nm}$ and a background refractive index of 1.33 mimicking the aqueous solution measurement. Both the far-field extinction cross-sectional spectra and the near electric field distributions were recorded from the simulations. Details of the simulation method used are described in our previous publications. ${ }^{51,53-56}$

\section{ASSOCIATED CONTENT}

\section{Supporting Information}

The Supporting Information is available free of charge on the ACS Publications website at DOI: 10.1021/acsomega.8b01153.

Nanoparticles of TEM, SERS, and simulation characterization (PDF)

\section{AUTHOR INFORMATION}

\section{Corresponding Author}

*E-mail: niveen.khashab@kaust.edu.sa.

ORCID

Chen Li: 0000-0002-7917-7479

Zahra Fakhraai: 0000-0002-0597-9882

Basem Moosa: 0000-0002-2350-4100

Niveen M. Khashab: 0000-0003-2728-0666

Notes

The authors declare no competing financial interest.

\section{ACKNOWLEDGMENTS}

We thank King Abdullah University of Science and Technology (KAUST) and King Abdulaziz City of Science and Technology (KACST) for financial support.

\section{REFERENCES}

(1) Shen, W.; Lin, X.; Jiang, C.; Li, C.; Lin, H.; Huang, J.; Wang, S.; Liu, G.; Yan, X.; Zhong, Q.; Ren, B. Reliable Quantitative SERS Analysis Facilitated by Core-Shell Nanoparticles with Embedded Internal Standards. Angew. Chem., Int. Ed. 2015, 54, 7308-7312.

(2) Fleischmann, M.; Hendra, P. J.; McQuillan, A. J. Raman spectra of pyridine adsorbed at a silver electrode. Chem. Phys. Lett. 1974, 26, $163-166$.

(3) Albrecht, M. G.; Creighton, J. A. Anomalously intense Raman spectra of pyridine at a silver electrode. J. Am. Chem. Soc. 1977, 99, 5215-5217.

(4) Lim, D.-K.; Jeon, K.-S.; Hwang, J.-H.; Kim, H.; Kwon, S.; Suh, Y. D.; Nam, J.-M. Highly uniform and reproducible surface-enhanced Raman scattering from DNA-tailorable nanoparticles with $1-\mathrm{nm}$ interior gap. Nat. Nanotechnol. 2011, 6, 452-460.

(5) Nie, S.; Emory, S. R. Probing Single Molecules and Single Nanoparticles by Surface-Enhanced Raman Scattering. Science 1997, $275,1102-1106$. 
(6) Kneipp, K.; Wang, Y.; Kneipp, H.; Perelman, L. T.; Itzkan, I.; Dasari, R. R.; Feld, M. S. Single Molecule Detection Using SurfaceEnhanced Raman Scattering (SERS). Phys. Rev. Lett. 1997, 78, 16671670.

(7) Le Ru, E. C.; Meyer, M.; Etchegoin, P. G. Proof of SingleMolecule Sensitivity in Surface Enhanced Raman Scattering (SERS) by Means of a Two-Analyte Technique. J. Phys. Chem. B 2006, 110, 1944-1948.

(8) Quyen, T. T. B.; Su, W.-N.; Chen, K.-J.; Pan, C.-J.; Rick, J.; Chang, C.-C.; Hwang, B.-J. Au@SiO2core/shell nanoparticle assemblage used for highly sensitive SERS-based determination of glucose and uric acid. J. Raman Spectrosc. 2013, 44, 1671-1677.

(9) Tan, E.-Z.; Yin, P.-G.; You, T.-t.; Wang, H.; Guo, L. Three Dimensional Design of Large-Scale $\mathrm{TiO} 2$ Nanorods Scaffold Decorated by Silver Nanoparticles as SERS Sensor for Ultrasensitive Malachite Green Detection. ACS Appl. Mater. Interfaces 2012, 4, 3432-3437.

(10) Kumar, A.; Kumar, S.; Rhim, W.-K.; Kim, G.-H.; Nam, J.-M. Oxidative nanopeeling chemistry-based synthesis and photodynamic and photothermal therapeutic applications of plasmonic core-petal nanostructures. J. Am. Chem. Soc. 2014, 136, 16317-16325.

(11) Sun, M.; Zhang, Z.; Zheng, H.; Xu, H. In-situ plasmon-driven chemical reactions revealed by high vacuum tip-enhanced Raman spectroscopy. Sci. Rep. 2012, 2, 647.

(12) Zheng, X.-S.; Hu, P.; Cui, Y.; Zong, C.; Feng, J.-M.; Wang, X.; Ren, B. BSA-coated nanoparticles for improved SERS-based intracellular pH sensing. Anal. Chem. 2014, 86, 12250-12257.

(13) Fang, Y.; Seong, N.-H.; Dlott, D. D. Measurement of the Distribution of Site Enhancements in Surface-Enhanced Raman Scattering. Science 2008, 321, 388-392.

(14) Chen, H.-Y.; Lin, M.-H.; Wang, C.-Y.; Chang, Y.-M.; Gwo, S. Large-Scale Hot Spot Engineering for Quantitative SERS at the Single-Molecule Scale. J. Am. Chem. Soc. 2015, 137, 13698-13705.

(15) Xu, L.; Yan, W.; Ma, W.; Kuang, H.; Wu, X.; Liu, L.; Zhao, Y.; Wang, L.; $\mathrm{Xu}, \mathrm{C}$. SERS encoded silver pyramids for attomolar detection of multiplexed disease biomarkers. Adv. Mater. 2015, 27, 1706-1711.

(16) Liu, Z.; Ding, S.-Y.; Chen, Z.-B.; Wang, X.; Tian, J.-H.; Anema, J. R.; Zhou, X.-S.; Wu, D.-Y.; Mao, B.-W.; Xu, X.; Ren, B.; Tian, Z.-Q. Revealing the molecular structure of single-molecule junctions in different conductance states by fishing-mode tip-enhanced Raman spectroscopy. Nat. Commun. 2011, 2, 305.

(17) Qian, X.-M.; Nie, S. M. Single-molecule and single-nanoparticle SERS: from fundamental mechanisms to biomedical applications. Chem. Soc. Rev. 2008, 37, 912-920.

(18) Zou, S.; Ma, L.; Li, J.; Xie, Z.; Zhao, D.; Ling, Y.; Zhang, Z. Quantification of trace chemicals in unknown complex systems by SERS. Talanta 2018, 186, 452-458.

(19) Zou, S.; Hou, M.; Li, J.; Ma, L.; Zhang, Z. Semi-quantitative analysis of multiple chemical mixtures in solution at trace level by surface-enhanced Raman Scattering. Sci. Rep. 2017, 7, 6186.

(20) Hou, M.; Huang, Y.; Ma, L.; Zhang, Z. Compositional Analysis of Ternary and Binary Chemical Mixtures by Surface-Enhanced Raman Scattering at Trace Levels. Nanoscale Res. Lett. 2015, 10, 437.

(21) Bell, S. E. J.; Sirimuthu, N. M. S. Quantitative surface-enhanced Raman spectroscopy. Chem. Soc. Rev. 2008, 37, 1012-1024.

(22) Bell, S. E. J.; Sirimuthu, N. M. S. Quantitative surface-enhanced Raman spectroscopy. Chem. Soc. Rev. 2008, 37, 1012-1024.

(23) Chen, L.; Choo, J. Recent advances in surface-enhanced Raman scattering detection technology for microfluidic chips. Electrophoresis 2008, 29, 1815-1828.

(24) Kämmer, E.; Olschewski, K.; Bocklitz, T.; Rösch, P.; Weber, K.; Cialla, D.; Popp, J. A new calibration concept for a reproducible quantitative detection based on SERS measurements in a microfluidic device demonstrated on the model analyte adenine. Phys. Chem. Chem. Phys. 2014, 16, 9056-9063.

(25) Zhu, C.; Meng, G.; Zheng, P.; Huang, Q.; Li, Z.; Hu, X.; Wang, X.; Huang, Z.; Li, F.; Wu, N. A Hierarchically Ordered Array of Silver-
Nanorod Bundles for Surface-Enhanced Raman Scattering Detection of Phenolic Pollutants. Adv. Mater. 2016, 28, 4871-4876.

(26) Zhou, N.; Meng, G.; Zhu, C.; Chen, B.; Qitao, Z.; Ke, Y.; Huo, D. A Silver-Grafted Sponge as an Effective Surface-Enhanced Raman Scattering Substrate. Sens. Actuators, B 2018, 258, 56-63.

(27) Kasera, S.; Biedermann, F.; Baumberg, J. J.; Scherman, O. A.; Mahajan, S. Quantitative SERS Using the Sequestration of Small Molecules Inside Precise Plasmonic Nanoconstructs. Nano Lett. 2012, 12, 5924-5928.

(28) Shen, W.; Lin, X.; Jiang, C.; Li, C.; Lin, H.; Huang, J.; Wang, S.; Liu, G.; Yan, X.; Zhong, Q.; Ren, B. Reliable Quantitative SERS Analysis Facilitated by Core-Shell Nanoparticles with Embedded Internal Standards. Angew. Chem., Int. Ed. 2015, 54, 7308-7312.

(29) Kang, J. W.; So, P. T. C.; Dasari, R. R.; Lim, D.-K. High resolution live cell Raman imaging using subcellular organelletargeting SERS-sensitive gold nanoparticles with highly narrow intra-nanogap. Nano Lett. 2015, 15, 1766-1772.

(30) Song, J.; Duan, B.; Wang, C.; Zhou, J.; Pu, L.; Fang, Z.; Wang, P.; Lim, T. T.; Duan, H. SERS-encoded nanogapped plasmonic nanoparticles: growth of metallic nanoshell by templating redox-active polymer brushes. J. Am. Chem. Soc. 2014, 136, 6838-6841.

(31) Guerrini, L.; Graham, D. Molecularly-mediated assemblies of plasmonic nanoparticles for Surface-Enhanced Raman Spectroscopy applications. Chem. Soc. Rev. 2012, 41, 7085-7107.

(32) Zhang, Y.; Yang, P.; Muhammed, M. A. H.; Moosa, B.; Alsaiari, S. K.; Almalik, A.; Kumar, A.; Ringe, E.; Khashab, N. M. Tunable and Linker Free Nanogaps in Core-Shell Plasmonic Nanorods for Selective and Quantitative Detection of Circulating Tumor Cells by SERS. ACS Appl. Mater. Interfaces 2017, 9, 37597-37605.

(33) Kim, M.; Ko, S. M.; Kim, J.-M.; Son, J.; Lee, C.; Rhim, W.-K.; Nam, J.-M. Dealloyed Intra-Nanogap Particles with Highly Robust, Quantifiable Surface-Enhanced Raman Scattering Signals for Biosensing and Bioimaging Applications. ACS Cent. Sci. 2018, 4, 277-287.

(34) Lin, L.; Zapata, M.; Xiong, M.; Liu, Z.; Wang, S.; Xu, H.; Borisov, A. G.; Gu, H.; Nordlander, P.; Aizpurua, J.; Ye, J. Nanooptics of Plasmonic Nanomatryoshkas: Shrinking the Size of a Core-Shell Junction to Subnanometer. Nano Lett. 2015, 15, 6419-6428.

(35) Bardhan, R.; Mukherjee, S.; Mirin, N. A.; Levit, S. D.; Nordlander, P.; Halas, N. J. Nanosphere-in-a-Nanoshell: A Simple Nanomatryushka. J. Phys. Chem. C 2010, 114, 7378-7383.

(36) Wang, Y.; Serrano, A. B.; Sentosun, K.; Bals, S.; Liz-Marzán, L. M. Stabilization and Encapsulation of Gold Nanostars Mediated by Dithiols. Small 2015, 11, 4314-4320.

(37) Abalde-Cela, S.; Aldeanueva-Potel, P.; Mateo-Mateo, C.; Rodríguez-Lorenzo, L.; Alvarez-Puebla, R. A.; Liz-Marzán, L. M. Surface-enhanced Raman scattering biomedical applications of plasmonic colloidal particles. J. R. Soc., Interface 2010, 7, S435-S450.

(38) Li, M.; Cushing, S. K.; Zhang, J.; Lankford, J.; Aguilar, Z. P.; $\mathrm{Ma}, \mathrm{D}$.; $\mathrm{Wu}, \mathrm{N}$. Shape-dependent surface-enhanced Raman scattering in gold-Raman-probe-silica sandwiched nanoparticles for biocompatible applications. Nanotechnology 2012, 23, 115501.

(39) Shanmukh, S.; Jones, L.; Driskell, J.; Zhao, Y.; Dluhy, R.; Tripp, R. A. Rapid and Sensitive Detection of Respiratory Virus Molecular Signatures Using a Silver Nanorod Array SERS Substrate. Nano Lett. 2006, 6, 2630-2636.

(40) Li, M.; Cushing, S. K.; Zhang, J.; Suri, S.; Evans, R.; Petros, W. P.; Gibson, L. F.; Ma, D.; Liu, Y.; Wu, N. Three-Dimensional Hierarchical Plasmonic Nano-Architecture Enhanced Surface-Enhanced Raman Scattering Immunosensor for Cancer Biomarker Detection in Blood Plasma. ACS Nano 2013, 7, 4967-4976.

(41) Li, M.; Zhang, J.; Suri, S.; Sooter, L. J.; Ma, D.; Wu, N. Detection of Adenosine Triphosphate with an Aptamer Biosensor Based on Surface-Enhanced Raman Scattering. Anal. Chem. 2012, 84, 2837-2842.

(42) Li, M.; Gou, H.; Al-Ogaidi, I.; Wu, N. Nanostructured Sensors for Detection of Heavy Metals: A Review. ACS Sustainable Chem. Eng. 2013, 1, 713-723.

(43) Black, K. C. L.; Yi, J.; Rivera, J. G.; Zelasko-Leon, D. C.; Messersmith, P. B. Polydopamine-enabled surface functionalization of 
gold nanorods for cancer cell-targeted imaging and photothermal therapy. Nanomedicine 2013, 8, 17.

(44) Lee, H.; Dellatore, S. M.; Miller, W. M.; Messersmith, P. B. Mussel-Inspired Surface Chemistry for Multifunctional Coatings. Science 2007, 318, 426.

(45) Lee, Y. S.; Bae, J. Y.; Koo, H. Y.; Lee, Y. B.; Choi, W. S. A remote-controlled generation of gold@polydopamine (core@shell) nanoparticles via physical-chemical stimuli of polydopamine/gold composites. Sci. Rep. 2016, 6, 22650.

(46) Lee, Y.; Lee, S. H.; Kim, J. S.; Maruyama, A.; Chen, X.; Park, T. G. Controlled synthesis of PEI-coated gold nanoparticles using reductive catechol chemistry for siRNA delivery. J. Controlled Release 2011, 155, 3-10.

(47) Zhang, Y.; Gu, C.; Schwartzberg, A. M.; Chen, S.; Zhang, J. Z. Optical trapping and light-induced agglomeration of gold nanoparticle aggregates. Phys. Rev. B: Condens. Matter Mater. Phys. 2006, 73, 165405.

(48) Xu, J.; Du, J.; Jing, C.; Zhang, Y.; Cui, J. Facile detection of polycyclic aromatic hydrocarbons by a surface-enhanced Raman scattering sensor based on the Au coffee ring effect. ACS Appl. Mater. Interfaces 2014, 6, 6891-6897.

(49) Xie, Y.; Wang, X.; Han, X.; Xue, X.; Ji, W.; Qi, Z.; Liu, J.; Zhao, B.; Ozaki, Y. Sensing of polycyclic aromatic hydrocarbons with cyclodextrin inclusion complexes on silver nanoparticles by surfaceenhanced Raman scattering. Analyst 2010, 135, 1389-1394.

(50) Stiles, P. L.; Dieringer, J. A.; Shah, N. C.; Van Duyne, R. P. Surface-Enhanced Raman Spectroscopy. Annu. Rev. Anal. Chem. 2008, 1, 601-626.

(51) Qian, Z.; Li, C.; Fakhraai, Z.; Park, S.-J. Unusual Weak Interparticle Distance Dependence in Raman Enhancement from Nanoparticle Dimers. J. Phys. Chem. C 2016, 120, 1824-1830.

(52) Gersten, J.; Nitzan, A. Electromagnetic theory of enhanced Raman scattering by molecules adsorbed on rough surfaces. J. Chem. Phys. 1980, 73, 3023-3037.

(53) Sanchez-Gaytan, B. L.; Swanglap, P.; Lamkin, T. J.; Hickey, R. J.; Fakhraai, Z.; Link, S.; Park, S.-J. Spiky Gold Nanoshells: Synthesis and Enhanced Scattering Properties. J. Phys. Chem. C 2012, 116, 10318-10324.

(54) Hastings, S. P.; Swanglap, P.; Qian, Z.; Fang, Y.; Park, S.-J.; Link, S.; Engheta, N.; Fakhraai, Z. Quadrupole-Enhanced Raman Scattering. ACS Nano 2014, 8, 9025-9034.

(55) Hastings, S. P.; Qian, Z.; Swanglap, P.; Fang, Y.; Engheta, N.; Park, S.-J.; Link, S.; Fakhraai, Z. Modal interference in spiky nanoshells. Opt. Express 2015, 23, 11290-11311.

(56) Qian, Z.; Hastings, S. P.; Li, C.; Edward, B.; McGinn, C. K.; Engheta, N.; Fakhraai, Z.; Park, S.-J. Raspberry-like Metamolecules Exhibiting Strong Magnetic Resonances. ACS Nano 2015, 9, 12631270. 